Arab Univ. J. Agric. Sci., Ain Shams Univ., Cairo, Egypt

28(4), 1271-1281, 2020

Website: http://ajs.journals.ekb.eg

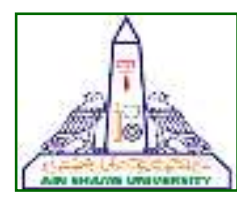

1271

\title{
Reproductive and Population Parameters of Empoasca Decipiens Paoli (Hemiptera: Cicadellidae) on Different Host Plants
}

\author{
Adnan AE Darwish ${ }^{\star}$ \\ Plant Protection Department, Faculty of Agriculture, Damanhour University, \\ Damanhour, Egypt
}

*Corresponding author: adnandarwish2012@yahoo.com

Received 3 October, 2020

Accepted 2 December, 2020

\section{Abstract}

Knowing the reproductive and population parameters (life table parameters) of a particular insect pest is very essential to develop its integrated pest management strategy. In this study, life table parameters of the leafhopper, Empoasca decipiens Paoli (Hemiptera - Cicadellidae) were calculated on four different host plants viz., potato (Solanum tuberosum L.), common bean (Phaseolus vulgaris L.), cotton (Gossypium barbadense L.) and zucchini squash (Cucurbita pepo L.). The results showed that, the net reproduction rates $\left(R_{0}\right)$ were 18.77 , 38.3, 30.63 and 27.27 offspring/ female / generation on cotton, common bean, potato and zucchini squash, respectively. The intrinsic rates of increase $\left(r_{\mathrm{m}}\right)$ were $0.15,0.19,0.149$ and 0.186 female $^{-1}$ day $^{-}$ ${ }^{1}$ with finite rates of increase $(\lambda)$ of $1.16,1.21,1.16$ and 1.21 females / female / day on cotton, common bean, potato and zucchini squash, respectively. The values of both of mean generation time $(T)$ and corrected generation time $\left(T_{c}\right)$ were highest for leafhopper insect reared on potato plant compared with those which reared on other three host plants. While the require times to duplicate the population (TD) were 4.6041, 3.6597, 4.661 and 3.727 days on cotton, common bean, potato and zucchini squash, respectively. The annual rates of increase (ARI) of $E$. decipiens were obtained as $7.28 \times 10^{23}, 1.05 \times 10^{30}$, $3.71 \times 10^{23}$ and $3.03 \times 10^{29}$ and it further reveal that the population would multiply 23, 30, 23 and 29 times in a year on each of cotton, common bean, potato and zucchini squash, respectively. On another hand, the calculated birth rates were 0.185 , $0.225,0.171$ and 0.224 and the intrinsic death rates were $0.0347,0.035,0.022$ and 0.038 in the leafhopper reared on the four host plants cotton, common bean, potato and zucchini squash, respectively. The
\end{abstract}

mean durations (day) of $E$. decipiens nymphal instars on the four different hosts also were studied. The duration of nymphal instars was significantly different from host plant to another. The nymphal stage of $E$. decipiens was completed in 11.04 , $13.75,12.28$ and 13.37 days on common bean, cotton, squash and potato, respectively.

Keywords: Empoasca decipiens, Life table, Net reproduction rates, Intrinsic rates

\section{Introduction}

The leafhopper, Empoasca decipiens Paoli (Hemiptera- Cicadellidae) is one of the most destructive sucking insect pests of vegetable and ornamental crops throughout the world. It has a wide range of plant hosts, short generation time and ability to transmit important plant viruses. The nymphs and adults of $E$. decipiens suck the plant sap by using its piercing-sucking mouthparts, injects it toxic saliva into leaves and cause Phytotoxicity (phytotoxic symptoms). Feeding injury of $E$. decipiens can result in discoloration, sometimes called "hopper burn" and consequently yield loss (Nault and Ammar 1989, Atwal and Singh 1990, Singh et al 2008, Mahmoud et al 2011, Khalafallah et al 2015, Darwish 2018). The green leafhopper usually colonized on the leaves undersurface of its plant hosts. The females of $E$. decipiens lay their eggs within the tissue of leaf vein (Raupach et al 2002, Backus et al 2005). In general, the host plants of a particular insect pest have a major effect on the biological aspects of this pest (Kim and Lee 2002, Bullas-appleton et al 2004, Naseri et al 2009, Khalafallah et al 2015). Also, many authors have reported that the differences in plant hosts of the insect pests may play an important role in it's population dynamic (Lu 
and $\mathrm{Xu}$ 1998). The life cycle parameters of the green leafhopper have been studied by different authors under different condition (Raupach et al 2002) and different hosts (Medeiros et al 2005, Naseri et al 2007, Fathi et al 2009). The basic knowledge of the pest's population dynamics is required for development of an adequate control strategy for any insect pest. Life tables are a fundamental tool for understanding the population dynamics of an insect pest (Pascua and Pascua 2002). The life table parameters such as mean generation time, gross reproduction rate and net reproduction rate are considered useful indices for comparing and/or predict the population growth potential of different insect pests under different environmental conditions such as food quality or host plants (Southwood 1966). Keeping in view the above mentioned information, this investigation was undertaken to evaluate the effect of host plants on life table parameters of Empoasca decipiens Paoli (Hemiptera: Cicadellidae).

\section{Materials and Methods}

\subsection{Stock cultures of E. decipiens}

To establish a stock culture of E. decipiens, the adults were collected using sweep net (or an aspirator) from unsprayed fields of four plant hosts (potato, common bean, cotton and zucchini squash). The collected adults were used to start stock colony for the present study. The insects were transferred with a fine hair brush after a temporary anesthetized for $5 \mathrm{~min}$ at $-4^{\circ} \mathrm{C}$ and reared on seedlings (at $2^{\text {nd }}$ and $3^{\text {rd }}$ leaf stage) cultivated individually in plastic pots. The adults that collected from a specific crop plants were rearing on seedlings of the same crop. Each seedlings species were separately placed inside wire breeding chamber (semi-field condition). Therefore, prior to the experiment the offspring of $E$. decipiens were reared at least for two generations on the above mentioned plants.

\subsection{Plant hosts}

Four plants species (represent four different families) namely potato (Solanum tuberosum L., variety Cara), common bean (Phaseolus vulgaris $\mathrm{L}$. variety Nebraska), cotton (Gossypium barbadense L. variety Ashmouni) and zucchini squash (Cucurbita pepo L. variety Eskandrani) were used in this study. The plants were individually cultivated in plastic pots placed inside wire breeding chamber. No insecticides were applied to the plants.
2.3 The life table parameters of $E$. decipiens on different hosts

The life table parameters of E. decipiens on the above mention plant hosts were studied by confining 20 pairs (one day old adult) of the insect with the leaves of 20 seedlings (1-2 month old plants) of each host using clip cages as described by Costa et al (1991). The females were allowed to lay eggs for 24 hour. The clip cages with the same pairs of $E$. decipiens were moved to another leaf. Until the last individual from each treatment was died, each part of leaf harbouring eggs (leaf disc) was examined by microscope to determine the daily egg numbers. Life tables were constructed using the following parameters following the method by Birch (1948) Southwood (1978), Carey (1993), Carey (1995) and Price (1997).

$$
\begin{aligned}
& \text { Pf or GRR }=\sum_{x=0}^{\infty} \mathbf{m}_{x} \\
& \mathbf{R}_{\mathbf{0}}=\sum_{x=\mathbf{0}}^{\infty} \mathbf{i}_{\mathbf{x}} \mathbf{m}_{\mathbf{x}} \\
& \mathbf{A E}=\frac{\sum_{x=0}^{\infty} \mathbf{m}_{\mathbf{x}} \mathbf{I}_{\mathbf{x}}}{\sum_{x=0}^{\infty} \mathbf{I}_{\mathbf{x}}} \\
& \text { AFs }=\frac{\sum_{x=0}^{\infty} \mathbf{x} \mathbf{m}_{\mathbf{x}}}{\sum_{x=0}^{\infty} \mathbf{m}_{\mathbf{x}}} \\
& \mathbf{T}=\frac{\sum_{x=0}^{\infty} \mathbf{x} \mathbf{i}_{\mathbf{x}} \mathbf{m}_{\mathbf{x}}}{\sum_{x=0}^{\infty} \mathbf{i}_{\mathbf{x}} \mathbf{m}_{\mathbf{x}}} \\
& \mathbf{T}_{\mathrm{c}}=\frac{\operatorname{In}(\mathbf{R o})}{\mathbf{R m}} \\
& r_{m}=\frac{\underset{\text { In }(\mathbf{R o})}{T}}{\mathrm{~T}} \\
& \mathrm{DT}=\frac{\operatorname{In}(2)}{\mathrm{rm}} \\
& \lambda=e^{r m} \\
& W M=e^{7 r m} \text { or }=\left(e^{r m}\right)^{7} \\
& \text { ARI = Antilog e } \mathrm{e}^{\mathrm{rm} 365} \\
& H^{2}=\left(R_{0}\right)^{2} \\
& \mathbf{b}=\frac{1}{\sum_{x=0}^{\infty} e^{-\mathrm{rmx} I x}} \\
& d=b-r_{m}
\end{aligned}
$$

where $(\mathbf{x})$ is the age of individuals in days, $\left(I_{x}\right)$ is the surviving individuals at age $x$ (Proportion of original cohort surviving to each stage), $\left(m_{x}\right)$ is the expected reproductive of female at age $x,(P f$ or GRR) is the potential fecundity or the gross reproductive rate, $\left(R_{0}\right)$ is the net reproductive rates in days, $(A E)$ is the average egg / day, (AFs) is the mean age fecundity schedule, $(T)$ is the mean of generation time in days, $\left(T_{c}\right)$ is the corrected generation time in days, $\left(r_{m}\right)$ is the intrinsic rate of natural increase, (DT) is the doubling time in days, $(\lambda)$ is the finite capacity of increase, (WM) is the weekly multiplication rate, (ARI) 
(Hemiptera: Cicadellidae) on Different Host Plants

is the annual rate of increase, $\left(\mathrm{HF}_{2}\right)$ is the hypothetical $F_{2}$ females, (b) is the intrinsic instantaneous birth rate and $(d)$ is the intrinsic death rate.

\subsection{Biological parameters of nymphs of E. decip- iens}

The $2^{\text {nd }}$ experiment was planned to study the biological parameters of nymphs of $E$. decipiens on the above mentioned host plants (common bean, cotton and zucchini squash). For each host species 20 newly emerged nymphs were singly maintained in glass jars and supplied with fresh leaves of the specific host. Jars were covered with muslin cloth at top and kept at $25 \pm 1^{\circ} \mathrm{C}$ temperature and $65 \pm 5$ $\mathrm{RH}$. The nymphs were observed every 6 hours and the durations of nymphal instars were recorded. The obtained data were subjected to statistical analysis (ANOVA). Differences among the means were determine by least significant differences test and difference was considered $95 \%$ significant at ( $P$ value $\leq 0.05)$.

\section{Results}

Based on the data presented in Tables 1-4, the $1^{\text {st }}$ deposited egg of $E$. decipiens was observed in the $11^{\text {th }}, 8^{\text {th }}, 8^{\text {th }}, 10^{\text {th }}$ day on potato, zucchini squash, cotton and common bean, respectively. The parameters of population and reproductive of $E$. decipiens on four different hosts (extracted from Tables 1-4) are given in Table 5 . The potential fecundity (Pf) was 45.897, 47.32, 34.14 and 61.898 eggs / female, with a $R_{0}$ of $30.63,27.26825,18.76775$ and 38.3 offspring / female and average number of laid eggs / female / day of 1.113823, 1.236655, 0.926802 and 1.605886 in potato, zucchini squash, cotton and common bean, respectively. The average duration between the parent's birth to that of their progeny or the mean length of generation $(T)$ of $E$. decipiens can be arranged in a descending order as follows: potato (23.015 days) > common bean (19.245 days) $>$ cotton (18.121 days) > zucchini squash (17.772 days) with corrected generation values $\left(T_{C}\right)$ values of $23.013,19.247,18.83976$ and 17.7727 days. The $\mathrm{r}_{\mathrm{m}}$ values were $0.1487,0.186,0.15055$ and 0.1894 / female / day with a $\lambda$ of $1.1604,1.205,1.16258$ and 1.209 females / female / day for $E$. decipiens insects which fed on potato, zucchini squash, cotton and common bean, respectively. In another hand, the leafhopper $E$. decipiens had the capacity to double its population in 4.661 days, with a weekly multiplication rate (WM) of 2.8336 times on potato plant. Each of zucchini squash, cotton and common bean required $3.727,4.6041$, and 3.6597 days to double their populations with MW of $3.6797,2.8706$, and 3.7684 folds, respectively. The $\mathrm{HF}_{2}$ values in $\mathrm{F} 2$ generation were 352.228, 1466.89, 938.1969 and 743.5574 in cotton, common bean, potato and zucchini squash, respectively. The ARI of E. decipiens were obtained as $7.283 \times 10^{23}, 1.047 \times 10^{30}$, $3.708 \times 10^{23}$ and $3.028 \times 10^{29}$ and these values further reveal that the population would multiply $23,30,23$ and 29 times in a year on each of on cotton, common bean, potato and zucchini squash, respectively. Also, the results of time table of $E$. decipiens shows that the calculated birth rate was 0.185289 , $0.224865,0.170718$ and 0.223747 while the intrinsic death rate was $0.034739,0.035465,0.022018$ and 0.037747 in the four host plants cotton, common bean, potato and zucchini squash, respectively.

Data in Table 6 revealed that the mean durations (in days) of $E$. decipiens nymphal instars on different host plants. The duration of nymphal instars was significantly different from host plant to another. On common bean plants, the mean durations of the five nymphal stadia were 1.91, 2.02, $2.13,2.33$ and 2.65 days, respectively. These durations on cotton plant recorded 2.35, 2.48, 2.77, 2.89 and 3.23 , days respectively. While in squash the durations of the five stadia of E. decipiens were 2.09, $2.39,2.34,2.52$ and 2.86 days and finally in potato plants these durations were 2.29, 2.56, 2.77, 2.83 and 3.08 days, respectively. The nymphal stage of E. decipiens was completed in 11.04, 13.75, 12.28 and 13.37 days on common bean, cotton, squash and potato, respectively $(F=59.324, P=0.005)$. 
Table 1. Life table and age-specific fecundity of $E$. decipiens feeding on potato

\begin{tabular}{|c|c|c|c|c|c|c|c|c|}
\hline Age (Day) & $\mathbf{i}_{\mathbf{x}}$ & Eggs/one female & $m_{x}$ & $\mathbf{i}_{\mathbf{x}} \mathbf{m}_{\mathbf{x}}$ & $\mathbf{i}_{x} m_{x} x$ & $\mathbf{x m}_{\mathbf{x}}$ & $e^{-r m x}$ & $e^{-r m x} \mathbf{i}_{x}$ \\
\hline 1 & 1 & 0 & 0 & 0 & 0 & 0 & 0.86174665 & 0.861747 \\
\hline 2 & 1 & 0 & 0 & 0 & 0 & 0 & 0.742607 & 0.742607 \\
\hline 3 & 1 & 0 & 0 & 0 & 0 & 0 & 0.639939 & 0.639939 \\
\hline 4 & 1 & 0 & 0 & 0 & 0 & 0 & 0.551466 & 0.551466 \\
\hline 5 & 1 & 0 & 0 & 0 & 0 & 0 & 0.475224 & 0.475224 \\
\hline 6 & 1 & 0 & 0 & 0 & 0 & 0 & 0.409522 & 0.409522 \\
\hline 7 & 0.95 & 0 & 0 & 0 & 0 & 0 & 0.352905 & 0.335259 \\
\hline 8 & 0.95 & 0 & 0 & 0 & 0 & 0 & 0.304114 & 0.288909 \\
\hline 9 & 0.9 & 0 & 0 & 0 & 0 & 0 & 0.262069 & 0.235863 \\
\hline 10 & 0.9 & 0 & 0 & 0 & 0 & 0 & 0.225837 & \begin{tabular}{|l}
0.203254 \\
\end{tabular} \\
\hline 11 & 0.9 & 0.2 & 0.18 & 0.162 & 1.782 & 1.98 & 0.194615 & 0.175153 \\
\hline 12 & 0.9 & 0.35 & 0.315 & 0.2835 & 3.402 & 3.78 & 0.167709 & 0.150938 \\
\hline 13 & 0.9 & 0.6 & 0.54 & 0.486 & 6.318 & 7.02 & 0.144522 & 0.13007 \\
\hline 14 & 0.85 & 0.75 & 0.6375 & 0.541875 & 7.58625 & 8.925 & 0.124542 & 0.10586 \\
\hline 15 & 0.85 & 1.7 & 1.445 & 1.22825 & 18.42375 & 21.675 & 0.107323 & 0.091225 \\
\hline 16 & 0.8 & 1.15 & 0.92 & 0.736 & 11.776 & 14.72 & 0.092486 & 0.073988 \\
\hline 17 & 0.8 & 2.9 & 2.32 & 1.856 & 31.552 & 39.44 & 0.079699 & 0.063759 \\
\hline 18 & 0.75 & 2.8 & 2.1 & 1.575 & 28.35 & 37.8 & 0.06868 & 0.05151 \\
\hline 19 & 0.75 & 2.6 & 1.95 & 1.4625 & 27.7875 & 37.05 & 0.059185 & 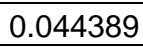 \\
\hline 20 & 0.75 & 3.25 & 2.4375 & 1.828125 & 36.5625 & 48.75 & 0.051003 & 0.038252 \\
\hline 21 & 0.75 & 3.5 & 2.625 & 1.96875 & 41.34375 & 55.125 & 0.043951 & 0.032963 \\
\hline 22 & 0.75 & 3.9 & 2.925 & 2.19375 & 48.2625 & 64.35 & 0.037875 & 0.028406 \\
\hline 23 & 0.7 & 3.95 & 2.765 & 1.9355 & 44.5165 & 63.595 & 0.032639 & 0.022847 \\
\hline 24 & 0.7 & 3.6 & 2.52 & 1.764 & 42.336 & 60.48 & 0.028126 & 0.019688 \\
\hline 25 & 0.7 & 4.4 & 3.08 & 2.156 & 53.9 & 77 & 0.024238 & 0.016966 \\
\hline 26 & 0.7 & 4.8 & 3.36 & 2.352 & 61.152 & 87.36 & 0.020887 & 0.014621 \\
\hline 27 & 0.65 & 4.15 & 2.6975 & 1.753375 & 47.34113 & 72.8325 & 0.017999 & 0.011699 \\
\hline 28 & 0.6 & 4.05 & 2.43 & 1.458 & 40.824 & 68.04 & 0.015511 & 0.009306 \\
\hline 29 & 0.6 & 3.9 & 2.34 & 1.404 & 40.716 & 67.86 & 0.013366 & 0.00802 \\
\hline 30 & 0.55 & 3.55 & 1.9525 & 1.073875 & 32.21625 & 58.575 & 0.011518 & 0.006335 \\
\hline 31 & 0.5 & 3.6 & 1.8 & 0.9 & 27.9 & 55.8 & 0.009926 & 0.004963 \\
\hline 32 & 0.4 & 3.15 & 1.26 & 0.504 & 16.128 & 40.32 & 0.008554 & 0.003421 \\
\hline 33 & 0.35 & 3.05 & 1.0675 & 0.373625 & 12.32963 & 35.2275 & 0.007371 & 0.00258 \\
\hline 34 & 0.35 & 2.25 & 0.7875 & 0.275625 & 9.37125 & 26.775 & 0.006352 & 0.002223 \\
\hline 35 & 0.3 & 1.8 & 0.54 & 0.162 & 5.67 & 18.9 & 0.005474 & 0.001642 \\
\hline 37 & 0.25 & 1.55 & 0.3875 & 0.096875 & 3.584375 & 14.3375 & 0.004065 & 0.001016 \\
\hline 38 & 0.25 & 1.2 & 0.3 & 0.075 & 2.85 & 11.4 & 0.003503 & 0.000876 \\
\hline 39 & 0.15 & 0.6 & 0.09 & 0.0135 & 0.5265 & 3.51 & 0.003019 & 0.000453 \\
\hline 40 & 0.1 & 0.55 & 0.055 & 0.0055 & 0.22 & 2.2 & 0.002601 & 0.00026 \\
\hline 41 & 0.1 & 0.4 & 0.04 & 0.004 & 0.164 & 1.64 & 0.002242 & 0.000224 \\
\hline 42 & 0.05 & 0.4 & 0.02 & 0.001 & 0.042 & 0.84 & 0.001932 & 9.66E-05 \\
\hline 43 & 0.05 & 0.2 & 0.01 & 0.0005 & 0.0215 & 0.43 & 0.001665 & 8.32E-05 \\
\hline \multirow[t]{2}{*}{44} & 0 & 0 & 0 & 0 & 0 & 0 & 0.001435 & 0 \\
\hline & & & $\begin{array}{c}\Sigma m_{x}= \\
45.8975\end{array}$ & $\begin{array}{c}\sum \mathrm{l}_{\mathrm{x}} \mathrm{m}_{\mathrm{x}}= \\
30.63013\end{array}$ & $\begin{array}{c}\Sigma 1_{x} m_{x} \mathrm{X}= \\
704.9554\end{array}$ & $\begin{array}{c}\sum m_{x} \mathrm{X}= \\
1107.738\end{array}$ & & \\
\hline
\end{tabular}


Reproductive and Population Parameters of Empoasca Decipiens Paoli (Hemiptera: Cicadellidae) on Different Host Plants

Table 2. Life table and age-specific fecundity of $E$. decipiens feeding on squash

\begin{tabular}{|c|c|c|c|c|c|c|c|c|}
\hline Age (Day) & $\mathbf{i}_{\mathbf{x}}$ & Eggs/one female & $m_{x}$ & $\mathbf{i}_{\mathbf{x}} \mathbf{m}_{\mathbf{x}}$ & $i_{x} m_{x} x$ & $\mathbf{x m}_{\mathrm{x}}$ & $e^{-r m x}$ & $e^{-r m x} i_{x}$ \\
\hline 1 & 1 & 0 & 0 & 0 & 0 & 0 & 0.830176 & 0.830176 \\
\hline 2 & 1 & 0 & 0 & 0 & 0 & 0 & 0.689192 & 0.689192 \\
\hline 3 & 1 & 0 & 0 & 0 & 0 & 0 & 0.572151 & 0.572151 \\
\hline 4 & 0.95 & 0 & 0 & 0 & 0 & 0 & 0.474986 & 0.451237 \\
\hline 5 & 0.95 & 0 & 0 & 0 & 0 & 0 & 0.394322 & 0.374606 \\
\hline 6 & 0.95 & 0 & 0 & 0 & 0 & 0 & 0.327357 & 0.310989 \\
\hline 7 & 0.9 & 0 & 0 & 0 & 0 & 0 & 0.271764 & 0.244587 \\
\hline 8 & 0.9 & 0.4 & 0.36 & 0.324 & 2.592 & 2.88 & 0.225612 & 0.20305 \\
\hline 9 & 0.85 & 1.15 & 0.9775 & 0.830875 & 7.477875 & 8.7975 & 0.187297 & 0.159203 \\
\hline 10 & 0.8 & 1.95 & 1.56 & 1.248 & 12.48 & 15.6 & 0.15549 & 0.124392 \\
\hline 11 & 0.8 & 3 & 2.4 & 1.92 & 21.12 & 26.4 & 0.129084 & 0.103267 \\
\hline 12 & 0.75 & 3 & 2.25 & 1.6875 & 20.25 & 27 & 0.107162 & 0.080372 \\
\hline 13 & 0.75 & 3.2 & 2.4 & 1.8 & 23.4 & 31.2 & 0.088964 & 0.066723 \\
\hline 14 & 0.7 & 3.35 & 2.345 & 1.6415 & 22.981 & 32.83 & 0.073855 & 0.051699 \\
\hline 15 & 0.7 & 3.5 & 2.45 & 1.715 & 25.725 & 36.75 & 0.061313 & 0.042919 \\
\hline 16 & 0.65 & 3.3 & 2.145 & 1.39425 & 22.308 & 34.32 & 0.050901 & 0.033085 \\
\hline 17 & 0.65 & 3.85 & 2.5025 & 1.626625 & 27.65263 & 42.5425 & 0.042256 & 0.027467 \\
\hline 18 & 0.65 & 4.15 & 2.6975 & 1.753375 & 31.56075 & 48.555 & 0.03508 & 0.022802 \\
\hline 19 & 0.6 & 4.05 & 2.43 & 1.458 & 27.702 & 46.17 & 0.029123 & 0.017474 \\
\hline 20 & 0.6 & 4.3 & 2.58 & 1.548 & 30.96 & 51.6 & 0.024177 & 0.014506 \\
\hline 21 & 0.55 & 4.2 & 2.31 & 1.2705 & 26.6805 & 48.51 & 0.020071 & 0.011039 \\
\hline 22 & 0.5 & 4.55 & 2.275 & 1.1375 & 25.025 & 50.05 & 0.016663 & 0.008331 \\
\hline 23 & 0.5 & 4.05 & 2.025 & 1.0125 & 23.2875 & 46.575 & 0.013833 & 0.006916 \\
\hline 24 & 0.45 & 4.1 & 1.845 & 0.83025 & 19.926 & 44.28 & 0.011484 & 0.005168 \\
\hline 25 & 0.45 & 4.2 & 1.89 & 0.8505 & 21.2625 & 47.25 & 0.009534 & 0.00429 \\
\hline 26 & 0.45 & 3.95 & 1.7775 & 0.799875 & 20.79675 & 46.215 & 0.007915 & 0.003562 \\
\hline 27 & 0.4 & 3.85 & 1.54 & 0.616 & 16.632 & 41.58 & 0.00657 & 0.002628 \\
\hline 28 & 0.35 & 3.9 & 1.365 & 0.47775 & 13.377 & 38.22 & 0.005455 & 0.001909 \\
\hline 29 & 0.3 & 3.7 & 1.11 & 0.333 & 9.657 & 32.19 & 0.004528 & 0.001358 \\
\hline 30 & 0.3 & 3.1 & 0.93 & 0.279 & 8.37 & 27.9 & 0.003759 & 0.001128 \\
\hline 31 & 0.25 & 3.25 & 0.8125 & 0.203125 & 6.296875 & 25.1875 & 0.003121 & 0.00078 \\
\hline 32 & 0.25 & 2.7 & 0.675 & 0.16875 & 5.4 & 21.6 & 0.002591 & 0.000648 \\
\hline 33 & 0.25 & 2.3 & 0.575 & 0.14375 & 4.74375 & 18.975 & 0.002151 & 0.000538 \\
\hline 34 & 0.2 & 2.1 & 0.42 & 0.084 & 2.856 & 14.28 & 0.001786 & 0.000357 \\
\hline 35 & 0.2 & 1.35 & 0.27 & 0.054 & 1.89 & 9.45 & 0.001482 & 0.000296 \\
\hline 36 & 0.2 & 1.1 & 0.22 & 0.044 & 1.584 & 7.92 & 0.001231 & 0.000246 \\
\hline 37 & 0.1 & 0.8 & 0.08 & 0.008 & 0.296 & 2.96 & 0.001022 & 0.000102 \\
\hline 38 & 0.1 & 0.7 & 0.07 & 0.007 & 0.266 & 2.66 & 0.000848 & 8.48E-05 \\
\hline 39 & 0.05 & 0.45 & 0.0225 & 0.001125 & 0.043875 & 0.8775 & 0.000704 & 3.52E-05 \\
\hline 40 & 0.05 & 0.2 & 0.01 & 0.0005 & 0.02 & 0.4 & 0.000585 & 2.92E-05 \\
\hline \multirow[t]{2}{*}{41} & 0 & 0 & 0 & 0 & 0 & 0 & 0.000485 & 0 \\
\hline & & & $\begin{array}{l}\Sigma m_{x}= \\
47.32\end{array}$ & $\begin{array}{c}\Sigma 1_{x} m_{x}= \\
27.26825\end{array}$ & $\begin{array}{c}\sum 1_{x} m_{x} x= \\
484.62\end{array}$ & $\begin{array}{c}\Sigma m_{x} x= \\
931.725\end{array}$ & & \\
\hline
\end{tabular}


Table 3. Life table and age-specific fecundity of $E$. decipiens feeding on common bean

\begin{tabular}{|c|c|c|c|c|c|c|c|c|}
\hline Age (Day) & $\mathbf{i}_{\mathbf{x}}$ & Eggs/one female & $\mathrm{m}_{\mathbf{x}}$ & $\mathbf{i}_{\mathbf{x}} \mathbf{m}_{\mathbf{x}}$ & $\mathbf{i}_{\mathbf{x}} \mathbf{m}_{\mathbf{x}} \mathbf{x}$ & $\mathrm{xm}_{\mathrm{x}}$ & $e^{-r m x}$ & $\mathrm{e}^{-\mathrm{rmx} \mathbf{i}_{\mathrm{x}}}$ \\
\hline 1 & 1 & 0 & 0 & 0 & 0 & 0 & 0.827356 & 0.827356 \\
\hline 2 & 1 & 0 & 0 & 0 & 0 & 0 & 0.684519 & 0.684519 \\
\hline 3 & 1 & 0 & 0 & 0 & 0 & 0 & 0.566341 & 0.566341 \\
\hline 4 & 1 & 0 & 0 & 0 & 0 & 0 & 0.468566 & 0.468566 \\
\hline 5 & 0.95 & 0 & 0 & 0 & 0 & 0 & 0.387671 & 0.368287 \\
\hline 6 & 0.95 & 0 & 0 & 0 & 0 & 0 & 0.320742 & 0.304705 \\
\hline 7 & 0.9 & 0 & 0 & 0 & 0 & 0 & 0.265368 & 0.238831 \\
\hline 8 & 0.85 & 0.55 & 0.4675 & 0.397375 & 3.179 & 3.74 & 0.219554 & 0.186621 \\
\hline 9 & 0.85 & 1.15 & 0.9775 & 0.830875 & 7.477875 & 8.7975 & 0.181649 & 0.154402 \\
\hline 10 & 0.85 & 2.25 & 1.9125 & 1.625625 & 16.25625 & 19.125 & 0.150289 & 0.127745 \\
\hline 11 & 0.8 & 2.45 & 1.96 & 1.568 & 17.248 & 21.56 & 0.124342 & 0.099474 \\
\hline 12 & 0.8 & 2.85 & 2.28 & 1.824 & 21.888 & 27.36 & 0.102875 & 0.0823 \\
\hline 13 & 0.75 & 2.2 & 1.65 & 1.2375 & 16.0875 & 21.45 & 0.085115 & 0.063836 \\
\hline 14 & 0.75 & 4 & 3 & 2.25 & 31.5 & 42 & 0.07042 & 0.052815 \\
\hline 15 & 0.75 & 4.25 & 3.1875 & 2.390625 & 35.85938 & 47.8125 & 0.058263 & 0.043697 \\
\hline 16 & 0.75 & 4.05 & 3.0375 & 2.278125 & 36.45 & 48.6 & 0.048204 & 0.036153 \\
\hline 17 & 0.7 & 4.65 & 3.255 & 2.2785 & 38.7345 & 55.335 & 0.039882 & 0.027917 \\
\hline 18 & 0.7 & 3.4 & 2.38 & 1.666 & 29.988 & 42.84 & 0.032996 & 0.023098 \\
\hline 19 & 0.7 & 4 & 2.8 & 1.96 & 37.24 & 53.2 & 0.0273 & 0.01911 \\
\hline 20 & 0.7 & 5.65 & 3.955 & 2.7685 & 55.37 & 79.1 & 0.022587 & 0.015811 \\
\hline 21 & 0.7 & 4.65 & 3.255 & 2.2785 & 47.8485 & 68.355 & 0.018687 & 0.013081 \\
\hline 22 & 0.6 & 4.05 & 2.43 & 1.458 & 32.076 & 53.46 & 0.015461 & 0.009277 \\
\hline 23 & 0.6 & 4.05 & 2.43 & 1.458 & 33.534 & 55.89 & 0.012792 & 0.007675 \\
\hline 24 & 0.55 & 5.2 & 2.86 & 1.573 & 37.752 & 68.64 & 0.010583 & 0.005821 \\
\hline 25 & 0.5 & 5.4 & 2.7 & 1.35 & 33.75 & 67.5 & 0.008756 & 0.004378 \\
\hline 26 & 0.5 & 5.5 & 2.75 & 1.375 & 35.75 & 71.5 & 0.007245 & 0.003622 \\
\hline 27 & 0.45 & 5.35 & 2.4075 & 1.083375 & 29.25113 & 65.0025 & 0.005994 & 0.002697 \\
\hline 28 & 0.45 & 5.55 & 2.4975 & 1.123875 & 31.4685 & 69.93 & 0.004959 & 0.002232 \\
\hline 29 & 0.45 & 5.75 & 2.5875 & 1.164375 & 33.76688 & 75.0375 & 0.004103 & 0.001846 \\
\hline 30 & 0.4 & 4.85 & 1.94 & 0.776 & 23.28 & 58.2 & 0.003395 & 0.001358 \\
\hline 31 & 0.4 & 3.85 & 1.54 & 0.616 & 19.096 & 47.74 & 0.002808 & 0.001123 \\
\hline 32 & 0.35 & 3.2 & 1.12 & 0.392 & 12.544 & 35.84 & 0.002324 & 0.000813 \\
\hline 33 & 0.3 & 2.6 & 0.78 & 0.234 & 7.722 & 25.74 & 0.001922 & 0.000577 \\
\hline 34 & 0.25 & 2.5 & 0.625 & 0.15625 & 5.3125 & 21.25 & 0.001591 & 0.000398 \\
\hline 35 & 0.2 & 2.7 & 0.54 & 0.108 & 3.78 & 18.9 & 0.001316 & 0.000263 \\
\hline 36 & 0.15 & 1.95 & 0.2925 & 0.043875 & 1.5795 & 10.53 & 0.001089 & 0.000163 \\
\hline 37 & 0.15 & 1.4 & 0.21 & 0.0315 & 1.1655 & 7.77 & 0.000901 & 0.000135 \\
\hline 38 & 0.05 & 0.85 & 0.0425 & 0.002125 & 0.08075 & 1.615 & 0.000745 & 3.73E-05 \\
\hline 39 & 0.05 & 0.55 & 0.0275 & 0.001375 & 0.053625 & 1.0725 & 0.000617 & 3.08E-05 \\
\hline \multirow[t]{2}{*}{40} & 0 & 0 & 0 & 0 & 0 & 0 & 0.00051 & 0 \\
\hline & & & $\begin{array}{c}\Sigma m_{x}= \\
61.8975\end{array}$ & $\begin{array}{c}\sum 1_{x} m_{x}= \\
38.30038\end{array}$ & $\begin{array}{c}\Sigma 1_{x} m_{x} X= \\
737.0894\end{array}$ & $\begin{array}{c}\sum m_{x} x= \\
1294.893\end{array}$ & & \\
\hline
\end{tabular}


Reproductive and Population Parameters of Empoasca Decipiens Paoli (Hemiptera: Cicadellidae) on Different Host Plants

Table 4. Life table and age-specific fecundity of $E$. decipiens feeding on cotton

\begin{tabular}{|c|c|c|c|c|c|c|c|c|}
\hline Age (Day) & $\mathbf{i}_{\mathbf{x}}$ & Eggs/one female & $m_{x}$ & $\mathbf{i}_{x} \mathbf{m}_{x}$ & $i_{x} m_{x} x$ & $\mathrm{xm}_{\mathrm{x}}$ & $e^{-r m x}$ & $e^{-r m x_{x_{x}}}$ \\
\hline 1 & 1 & 0 & 0 & 0 & 0 & 0 & 0.860153 & 0.860153 \\
\hline 2 & 1 & 0 & 0 & 0 & 0 & 0 & 0.739863 & 0.739863 \\
\hline 3 & 1 & 0 & 0 & 0 & 0 & 0 & 0.636395 & 0.636395 \\
\hline 4 & 0.95 & 0 & 0 & 0 & 0 & 0 & 0.547397 & 0.520027 \\
\hline 5 & 0.95 & 0 & 0 & 0 & 0 & 0 & 0.470845 & 0.447303 \\
\hline 6 & 0.9 & 0 & 0 & 0 & 0 & 0 & 0.404999 & 0.364499 \\
\hline 7 & 0.9 & 0 & 0 & 0 & 0 & 0 & 0.348361 & 0.313525 \\
\hline 8 & 0.85 & 0 & 0 & 0 & 0 & 0 & 0.299644 & 0.254697 \\
\hline 9 & 0.85 & 0 & 0 & 0 & 0 & 0 & 0.257739 & 0.219079 \\
\hline 10 & 0.8 & 0.35 & 0.28 & 0.224 & 2.24 & 2.8 & 0.221695 & 0.177356 \\
\hline 11 & 0.8 & 1.05 & 0.84 & 0.672 & 7.392 & 9.24 & 0.190692 & 0.152553 \\
\hline 12 & 0.8 & 2.15 & 1.72 & 1.376 & 16.512 & 20.64 & 0.164024 & 0.131219 \\
\hline 13 & 0.75 & 2.95 & 2.2125 & 1.659375 & 21.57188 & 28.7625 & 0.141086 & 0.105814 \\
\hline 14 & 0.75 & 2.25 & 1.6875 & 1.265625 & 17.71875 & 23.625 & 0.121355 & 0.091017 \\
\hline 15 & 0.7 & 2.55 & 1.785 & 1.2495 & 18.7425 & 26.775 & 0.104384 & 0.073069 \\
\hline 16 & 0.65 & 2.95 & 1.9175 & 1.246375 & 19.942 & 30.68 & 0.089786 & 0.058361 \\
\hline 17 & 0.65 & 3.95 & 2.5675 & 1.668875 & 28.37088 & 43.6475 & 0.07723 & 0.0502 \\
\hline 18 & 0.65 & 3.05 & 1.9825 & 1.288625 & 23.19525 & 35.685 & 0.06643 & 0.043179 \\
\hline 19 & 0.6 & 3.05 & 1.83 & 1.098 & 20.862 & 34.77 & 0.05714 & 0.034284 \\
\hline 20 & 0.55 & 3.15 & 1.7325 & 0.952875 & 19.0575 & 34.65 & 0.049149 & 0.027032 \\
\hline 21 & 0.5 & 5.05 & 2.525 & 1.2625 & 26.5125 & 53.025 & 0.042275 & 0.021138 \\
\hline 22 & 0.45 & 5.45 & 2.4525 & 1.103625 & 24.27975 & 53.955 & 0.036363 & 0.016364 \\
\hline 23 & 0.45 & 4.35 & 1.9575 & 0.880875 & 20.26013 & 45.0225 & 0.031278 & 0.014075 \\
\hline 24 & 0.4 & 4.05 & 1.62 & 0.648 & 15.552 & 38.88 & 0.026904 & 0.010762 \\
\hline 25 & 0.4 & 4.75 & 1.9 & 0.76 & 19 & 47.5 & 0.023141 & 0.009257 \\
\hline 26 & 0.35 & 4.15 & 1.4525 & 0.508375 & 13.21775 & 37.765 & 0.019905 & 0.006967 \\
\hline 27 & 0.3 & 3.95 & 1.185 & 0.3555 & 9.5985 & 31.995 & 0.017122 & 0.005136 \\
\hline 28 & 0.25 & 3.15 & 0.7875 & 0.196875 & 5.5125 & 22.05 & 0.014727 & 0.003682 \\
\hline 29 & 0.25 & 2.25 & 0.5625 & 0.140625 & 4.078125 & 16.3125 & 0.012668 & 0.003167 \\
\hline 30 & 0.2 & 2.05 & 0.41 & 0.082 & 2.46 & 12.3 & 0.010896 & 0.002179 \\
\hline 31 & 0.2 & 2.25 & 0.45 & 0.09 & 2.79 & 13.95 & 0.009372 & 0.001874 \\
\hline 32 & 0.15 & 1.35 & 0.2025 & 0.030375 & 0.972 & 6.48 & 0.008062 & 0.001209 \\
\hline 33 & 0.1 & 0.5 & 0.05 & 0.005 & 0.165 & 1.65 & 0.006934 & 0.000693 \\
\hline 34 & 0.1 & 0.25 & 0.025 & 0.0025 & 0.085 & 0.85 & 0.005964 & 0.000596 \\
\hline 35 & 0.05 & 0.1 & 0.005 & 0.00025 & 0.00875 & 0.175 & 0.00513 & 0.000257 \\
\hline \multirow[t]{2}{*}{36} & 0 & 0 & 0 & 0 & 0 & 0 & 0.004413 & 0 \\
\hline & & & $\begin{array}{l}\Sigma m_{x}= \\
34.14\end{array}$ & $\begin{array}{c}\Sigma 1_{x} m_{x}= \\
18.76775\end{array}$ & $\begin{array}{c}\Sigma 1_{x} m_{x} X= \\
340.0968\end{array}$ & $\begin{array}{c}\Sigma m_{x} x= \\
673.185\end{array}$ & & \\
\hline
\end{tabular}


Table 5. Life parameters of E. decipiens on four different plant hosts

\begin{tabular}{|c|c|c|c|c|c|c|}
\hline \multicolumn{2}{|r|}{ Parameter } & Formula & Cotton & $\begin{array}{c}\text { Common } \\
\text { bean }\end{array}$ & Potato & $\begin{array}{l}\text { Zucchini } \\
\text { squash }\end{array}$ \\
\hline \multirow{4}{*}{ 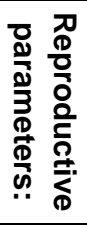 } & Potential fecundity (Pf) & $=\Sigma m_{x}$ & 34.14 & 61.898 & 45.897 & 47.32 \\
\hline & Net reproduction rate & $\mathrm{Ro}=\Sigma \mathrm{Ixm} x$ & 18.76775 & 38.3 & 30.63 & 27.26825 \\
\hline & Average eggs / day & $\Sigma \mathrm{I}_{\mathrm{x}} \mathrm{m}_{\mathrm{x}} / \Sigma \mathrm{I}_{\mathrm{x}}$ & 0.926802 & 1.605886 & 1.113823 & 1.236655 \\
\hline & $\begin{array}{l}\text { Mean age fecundity } \\
\text { schedule }\end{array}$ & $\Sigma \mathrm{xm}_{\mathrm{x}} / \Sigma \mathrm{m}_{\mathrm{x}}$ & 19.71837 & 20.91995 & 24.13503 & 19.68988 \\
\hline \multirow{10}{*}{ 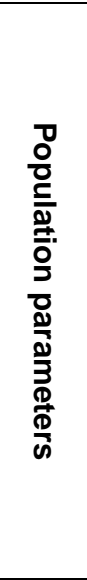 } & Mean generation time & $\begin{array}{c}\mathrm{T}=\Sigma \mathrm{Ixm}_{\mathrm{x}} \mathrm{x} / \Sigma \\
\mathrm{Ix}_{\mathrm{X}} \mathrm{m}\end{array}$ & 18.121 & 19.245 & 23.015 & 17.772 \\
\hline & $\begin{array}{l}\text { The intrinsic rate of } \\
\text { natural increase }\end{array}$ & $r_{m}=\ln R_{0} / T$ & 0.15055 & 0.1894 & 0.1487 & 0.186 \\
\hline & Finite rate of increase & $\lambda=e^{r m}$ & 1.16258 & 1.209 & 1.1604 & 1.205 \\
\hline & $\begin{array}{c}\text { Corrected generation } \\
\text { time }\end{array}$ & $\mathrm{T}_{\mathrm{c}}=\ln \mathrm{Ro} / \mathrm{r}_{\mathrm{m}}$ & 18.83976 & 19.247 & 23.013 & 17.7727 \\
\hline & Doubling time & $\mathrm{DT}=\ln 2 / \mathrm{r}_{\mathrm{m}}$ & & 3.6597 & 4.661 & 3.727 \\
\hline & \begin{tabular}{|c|} 
Weekly multiplication rate \\
(WM)
\end{tabular} & $e^{7 r m}$ or $\left(e^{r m}\right)^{7}$ & 2.8706 & 3.7684 & 2.8336 & 3.6797 \\
\hline & Intrinsic birth rate & $b=1 /\left.\Sigma e^{-r m x}\right|_{x}$ & 0.185289 & 0.224865 & 0.170718 & 0.223747 \\
\hline & Intrinsic death rate & $d=b-r_{m}$ & 0.034739 & 0.035465 & 0.022018 & 0.037747 \\
\hline & Annual Rate of Increase & Antilog $\mathrm{e}^{\mathrm{rm}} 365$ & $7.283 \times 10^{23}$ & $1.047 \times 10^{30}$ & $3.708 \times 10^{23}$ & $3.028 \times 10^{29}$ \\
\hline & Hypothetical F2 females & $\left(R_{0}\right)^{2}$ & 352.228 & 1466.89 & 938.1969 & 743.5574 \\
\hline
\end{tabular}

Table 6. Mean durations (in days) of the nymphal instars of E. decipiens on different host plants

\begin{tabular}{|c|c|c|c|c|c|c|}
\hline Hosts & $1^{\text {st }}$ instar & $2^{\text {nd }}$ instar & $3^{\text {rd }}$ instar & $4^{\text {th }}$ instar & $5^{\text {th }}$ instar & Total duration \\
\hline Common bean & $1.91 \pm 0.22^{\mathrm{d}}$ & $2.02 \pm 0.17^{c}$ & $2.13 \pm 0.23^{b}$ & $2.33 \pm 0.24^{c}$ & $2.65 \pm 0.28^{c}$ & $11.04 \pm 0.58^{c}$ \\
\hline Cotton & $2.35 \pm 0.2^{a}$ & $2.48 \pm 0.23^{a b}$ & $2.77 \pm 0.25^{\mathrm{a}}$ & $2.89 \pm 0.21^{a}$ & $3.23 \pm 0.22^{a}$ & $13.75 \pm 0.54^{a}$ \\
\hline Squash & $2.09 \pm 0.26^{c}$ & $2.39 \pm 0.19^{b}$ & $2.34 \pm 0.23^{b}$ & $2.52 \pm 0.21^{b c}$ & $2.86 \pm 0.28^{b c}$ & $12.28 \pm 0.58^{b}$ \\
\hline Potato & $2.29 \pm 0.26^{\mathrm{ab}}$ & $2.56 \pm 0.19^{a}$ & $2.77 \pm 0.31^{\mathrm{a}}$ & $2.83 \pm 0.25^{a}$ & $3.08 \pm 0.3^{\mathrm{ab}}$ & $13.37 \pm 0.24^{a}$ \\
\hline General mean & $2.15 \pm 0.29^{b c}$ & $2.34 \pm 0.29^{b}$ & $2.48 \pm 0.38^{b}$ & $2.62 \pm 0.32^{b}$ & $2.92 \pm 0.35^{b c}$ & $12.44 \pm 1.242^{b}$ \\
\hline F value & 11.815 & 22.579 & 20.682 & 15.870 & 9.368 & 59.324 \\
\hline L. S. D. & 0.17015 & 0.1556 & 0.2173 & 0.2044 & 0.28875 & 0.5367 \\
\hline
\end{tabular}

\section{Discussion}

Information of the biological parameters of a particular insect pest can vary from one host to another, and this factor is very essential to determine the suitability of the plants species as a host to various insect pests. The higher reproductive rates and short development time of an insect pest on a particular host plant reflect the suitability of this plant species as a host. (Van Lenteren and Noldus 1990, Awmack and Leather 2002, Saeed et al 2010). From the above-mentioned results, its obvious that the parameters of life table of Empoasca decipiens differed when reared in different hosts. The shorted pre-oviposition period for females of $E$. decipiens was observed in zucchini squash and cotton pants (8 days), while the longest pre-oviposition period was recorded at the potato plants ( 11 day). These results are slightly different with the results of Khalafallah et al (2015) who reported 6.76 and 6.5 days as a pre-oviposition period for $E$. decipiens in each of cotton and faba bean, respectively. In this study, the $\mathrm{R}_{0}$ of $E$. decipiens ranged from 18.77 in cotton to 38.3 in common bean plants, respectively. In similar results, Talebi et al (2010) found that the $\mathrm{R}_{0}$ of E. decipiens was $22.03,20.07,22.06$ and 22.31 in four cultivars of sugar beet namely, Shirin, Rasool, PP8 and IC, respectively. The $r_{m}$ varied from 0.1487 to 0.1894 in potato and common bean. The values of this parameter on the four different hosts are similar with those reported by Talebi et al (2010) who reported $0.099,0.103,0.102$ and $0.104 \mathrm{rm}$ for four different sugar beet cultivars. Also these results are in agreement with Tokuda and Matsumura (2004) 
(Hemiptera: Cicadellidae) on Different Host Plants

who reported 0.105 as a value of $r_{m}$ for Cicadulina bipunctata (Hemoptera: Cicadellidae) at $28.3^{\circ} \mathrm{C}$. Increase of the $r_{m}$ value on common bean can be attributed both to decline in generation time and to an increase in $R_{0}$ value. The decreasing on generation time was due to a reduction in the reproductive period. The fore-mentioned results confirm that common bean demonstrated higher degree of feed sources and preferred by the pest as indicated by $R_{0}, r_{m}$ and $\lambda$. The results of studying the nymphal instar durations clarify that the common bean plant was the most suitable host for E. decipiens followed by squash, potato and finally cotton plants. These results are in synchronize with the results of both of Raupach et al (2002) and Mahmoud et al (2011) who found that the broad bean was the most suitable host (short development time) and Khalafallah, et al (2015) who stated that the faba bean was most suitable than cotton for E. decipiens.

\section{Acknowledgment}

The author would like to thank the members of plant protection department, Faculty of Agriculture, Damanhour University for their efforts during these experiments

\section{References}

Atwal, AS; Singh, B (1990) Pest population and assessment of crop losses. ICAR Publication, Indian council of Agricultural Research, New Delhi, 265 pp.

Backus, EA; Serrano, MS; Ranger, CM (2005) Mechanism of Hopperburn: An Overview of Insect Taxonomy, Behavior and Physiology. Ann Rev Entomol, 50, 125-151.

Awmack, CS; Leather, SR (2002) Host plant quality and fecundity in herbivorous insects. Annual Review of Entomology 47, 817-844.

Birch, LC (1948) The intrinsic rate of natural increase of an insect population. J Anim Ecol 17, 1526.

Bullas-appleton, ES; Otis, G; Gillard, C; Schaafsma, AW (2004) Potato Leafhopper (Homoptera: Cicadellidae) Varietal Preferences in Edible Beans in Relation to Visual and Olfactory Cues. Environmental Entomology 33, 1381-1388.

Carey, JR (1993) Applied Demography for Biologists with Special Emphasis in Insects. Oxford University Press, New York, 206 pp.
Carey, JR (1995) Insect Demography. In: Encyclopedia of Environmental Biology, Academic Press, San Diego, pp. 289-303.

Costa, HS; Brown, JK; Byrne, DN (1991) Life history traits of the whitefly, Bemisia tabaci (Homoptera: Aleyrodidae) on six virus-infected or healthy plant species. Environmental Entomology 20, 1102-1107.

Darwish, AAE (2018) Variations in the susceptibility of some potato (Solanum tuberosum L.) cultivars to infestation with certain piercing sucking insect pests. Journal of Plant Protection and Pathology 9 , 849-853.

Fathi, SAA; Nouri-Ganbalani, G; Rafiee-Dastjerdi, H (2009) Life cycle parameters of Empoasca decipiens Paoli (Hom.: Cicadellidae) on four potato cultivars (Solanum tuberosum L.) in Iran. J Entomol 6, 96-101.

Khalafallah, EME; Khattab, MA; EL-Srand, EA (2015) Biology of Empoasca decipiens Paoli on Cotton and Faba Bean Plants. Middle East Journal of Agriculture Research 4, 503-508.

Kim, DS; Lee, JH (2002) Egg and larval survivorship of Carposina sasakii (Lepidoptera: Carposinidae) in apple and peach and their effects on adult population dynamics in orchards. Environ Entomol 31, 686-692.

Lu, ZQ; Xu, YH (1998) The consideration of the incessant outbreak of the cotton bollworm, Helicoverpa armigera. Entomol Knowledge 35, 132136.

Mahmoud, YA; Amr, EM; Ebadah, IMA (2011) Some ecological behaviors of the leafhopper, Empoasca decipiens (Paoli) on some winter plantations in Egypt. J Basic Appl Sci Res 1, 88-94.

Medeiros, AH; Delalibera, IJ; Tingey, WM (2005) Aspects of potato leafhopper (Homoptera: Cicadellidae) biology on Solanum berthaultii and other potato genotypes. J Econ Entomol 98, 1704-1709.

Nault, LR; Ammar, ED (1989) Leafhopper and plant hopper transmission of plant viruses. Annual Rev Ins 34, 301-329.

Naseri, B; Fathipour, Y; Talebi, AA (2007) Comparison of some biological aspects of Empoasca decipiens (Homoptera: Cicadellidae) on four bean species. J Entomol Soc Iran 27, 1-3.

Naseri, B; Fathipour, Y; Talebi, AA (2009) Population density and spatial distribution pattern of $\mathrm{Em}$ - 
poasca decipiens (Hemiptera: Cicadellidae) on different bean species. J Agric Sci Technol 11, 239248

Pascua, LT; Pascua, ME (2002) Life Table of Cotton Bollworm, Helicoverpa armigera Hübner (Lepidoptera: Noctuidae) in Batac, llocos Norte, Philippines. Philipp J Sci 131, 75-89.

Price, P (1997) Insect Ecology. New York, John Wiley \& Sons, 874 pp.

Raupach, K; Borgemeister, C; Hommes, M; Poehling, H; Sétamou, M (2002) Effect of temperature and host plants on the bionomics of Empoasca decipiens (Homoptera: Cicadellidae). Crop Protection 21, 113- 119.

Saeed, R; Sayyed, AH; Shad, SA; Zaka, SM (2010) Effect of different host plants on the fitness of diamond-back moth, Plutella xylostella (Lepidoptera: Plutellidae). Crop Protection 29, 178-182.

Singh, S; Choudhary, DP; Sharma, HC; Mahla, RS; Mathur, YS; Ahuja, DB (2008) Effect of insecticidal molecules against jassid and shoot and fruit borer in okra. Indian Journal of Entomology 70, 197-199.
Southwood, TRE (1966) Ecological Methods With Particular Reference To The Study of Insect Populations. Methuen, London, UK, 524 pp.

Southwood, TRE (1978) Ecological Methods With Particular Reference To The Study of Insect Populations. $2^{\text {nd }}$ edition. London: Chapman and Hall, 524 pp.

Talebi, AA; Izadpanah, A; Moharramipour, S; Fathipour, Y; Naseri, B (2010) Demographic study of the green leafhopper, Empoasca decipiens (Hemiptera: Cicadellidae) on four sugar beet cultivars. Appl Ent Phytopath 77, 1-13.

Tokuda, M; Matsumura, M (2004) Effect of temperature on the development and reproduction of the maize orange leafhopper Cicadulina bipunctata (Melichar) (Homoptera: Cicadellidae). Appl Entomol Zool 40, 213- 220.

Van Lenteren, JC; Noldus, LPJJ (1990) Whiteflyplant relationship: Behavioural and ecological aspects. In: Gerling D, ed. Whiteflies: Their bionomics, pest status and management. Hampshire, Intercept Ltd, pp 47-89. 


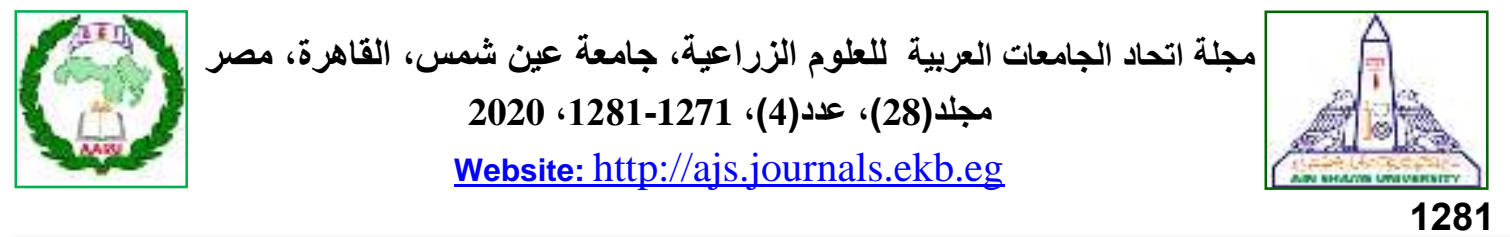

\section{Empoasca decipiens Paoli إحصائيات التكاثر والعثيرة لنطاط الاورلق علي عوائل نباتية مختلفة}

[90]

$$
\text { قسم وقاية النبات - كلية الزراعة - جامعة دمنهور - دمنهور - مصر الفيد درويش" عبداح }
$$

*Corresponding author: adnandarwish2012@yahoo.com

الحقيقية Intrinsic rates of increase سجلت 0,15 و 0,189 و 0,148 و 0,186/ انثي / يوم مع معدل

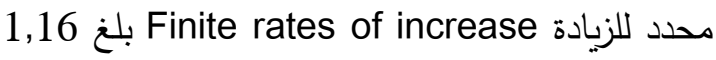

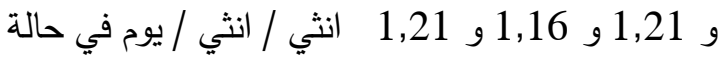
الحشرات التي تم تربيتها علي نباتات القطن والفاصوليا والبطاطس والكوسة، علي الترتيب. متوسط مدة الجيات الجيل ومدة الجيل المصححة كانت اعلي ما يمكن للحشرات

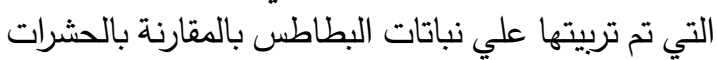

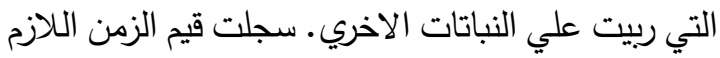

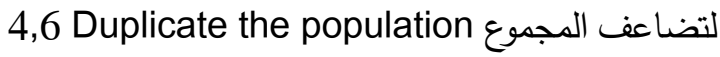
و 3,66 و 4,66 و 3,73 يوم للحشرات المرباه علي نباتات القطن والفاصوليا والبطاطس والكوسة، علي

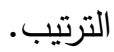

معرفة الإحصاءات البيولوجية (إحصاءات جداول

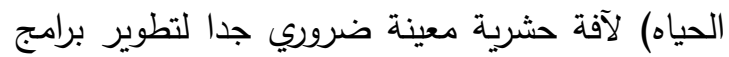

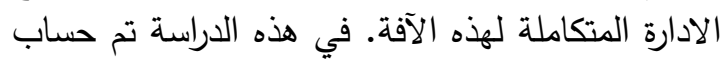

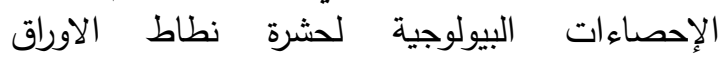
Empoasca decipiens مختلفة وهي نبات البطاطس، نبات الفاصوليا، نبات أبرات القطن، نبات الكوسة. أوضحت النتائج أن معدل التكاثر الصافي Net reproduction rate سجل أنس 38,3 و 30,63 و 27,27 فرد / أنثي / جيل في سلي

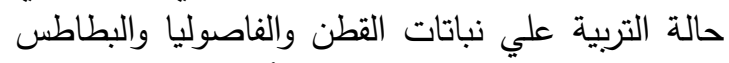
والكوسة، علي الترتيب. في حين أن قيم معدل الزيادة 